\title{
Emitter-Quencher Pair of Single Atomic Co Sites and Monolayer Titanium Carbide MXenes for Luminol Chemiluminescent Reaction
}

Hui Ouyang*, Jiaxin Xian, Shuai Luo, Lvxia Zhang, Wenwen Wang, and Zhifeng $\mathrm{Fu}^{*}$

Key Laboratory of Luminescence Analysis and Molecular Sensing (Ministry of Education)

College of Pharmaceutical Sciences

Southwest University

Chongqing, 400715, China

E-mail address: ouyanghui@swu.edu.cn; fuzf@swu.edu.cn

\section{Table of Contents}

Figure S1. SEM photograph of UiO-66 MOFs.

Figure S2. XPS patterns of Co SASCs: (A) survey and (B) Co 2p.

Figure S3. EDS spectrum of Co SASCs.

Figure S4. PXRD patterns of Co SASCs and UiO-66.

Figure S5. FTIR spectra of Co SASCs and UiO-66.

Figure S6. (A) Thermogravimetric analysis curve of Co SASCs measured in $\mathrm{N}_{2}$. (B) $\mathrm{N}_{2}$ adsorption/desorption isotherms of Co SASCs and UiO-66.

Figure S7. Quenching rates of different ROS scavengers on Co SASCs-enhanced luminol CL reaction: (A) ascorbic acid, (B) tryptophan, (C) tert-butyl alcohol and (D) superoxide dismutase $(n=3)$.

Figure S8. CL spectrum of Co SASCs-enhanced luminol- $\mathrm{H}_{2} \mathrm{O}_{2}$ reaction and absorption spectrum of $\mathrm{Ti}_{3} \mathrm{C}_{2}$ MXenes. 
Figure S9. (A) TEM and (B) AFM photographs of $\mathrm{Ti}_{3} \mathrm{C}_{2}$ MXenes.

Figure S10. CL signals from Co SASCs-enhanced luminol reaction quenched by graphene oxide or monolayer $\mathrm{Ti}_{3} \mathrm{C}_{2}$ MXenes $(n=3)$. Here the both quenchers were at $100 \mu \mathrm{g} \mathrm{mL}^{-1}$, and $\mathrm{H}_{2} \mathrm{O}$ was adopted as a control.

Figure S11. (A) Effect of $\mathrm{pH}$ value of the reaction medium on the CL signal from Co SASCsenhanced luminol reaction in the presence/absence of $\mathrm{Ti}_{3} \mathrm{C}_{2}$ Mxenes $(n=3)$. The concentration of luminol, $\mathrm{H}_{2} \mathrm{O}_{2}, \mathrm{Ti}_{3} \mathrm{C}_{2}$ MXenes and Co SASCs were $1.0 \times 10^{-6} \mathrm{M}, 0.1 \mathrm{M}, 50 \mu \mathrm{g} \mathrm{mL}^{-1}$, and 20 $\mu \mathrm{g} \mathrm{mL}-1$, respectively. (B) Effect of the amount of $\mathrm{Ti}_{3} \mathrm{C}_{2}$ MXenes-labeled tracer antibody on the CL signal for detecting cTnI at $7.5 \mathrm{pg} \mathrm{mL}^{-1}(n=3)$. (C) Effect of concentration of Co SASCs on the CL signal for detecting cTnI at $7.5 \mathrm{pg} \mathrm{mL}^{-1}(n=3)$. (D) Effect of migration time of $\mathrm{Ti}_{3} \mathrm{C}_{2}$ MXenes-labeled tracer antibody on the CL signal for detecting cTnI at $7.5 \mathrm{pg} \mathrm{mL}^{-1}(n=3)$.

Figure S12. CL signals produced by cTnI and the potential interferents all at $10 \mathrm{pg} \mathrm{mL}^{-1}(n=$ $3)$.

Table S1. Structural parameters of $\mathrm{Co}$ foil, $\mathrm{CoO}, \mathrm{Co}_{3} \mathrm{O}_{4}$, and $\mathrm{Co} \mathrm{SASC}$ extracted from the EXAFS fitting.

Table S2. Comparison of analytical performances from different quenching-type protocols for detecting cTnI.

\section{References}




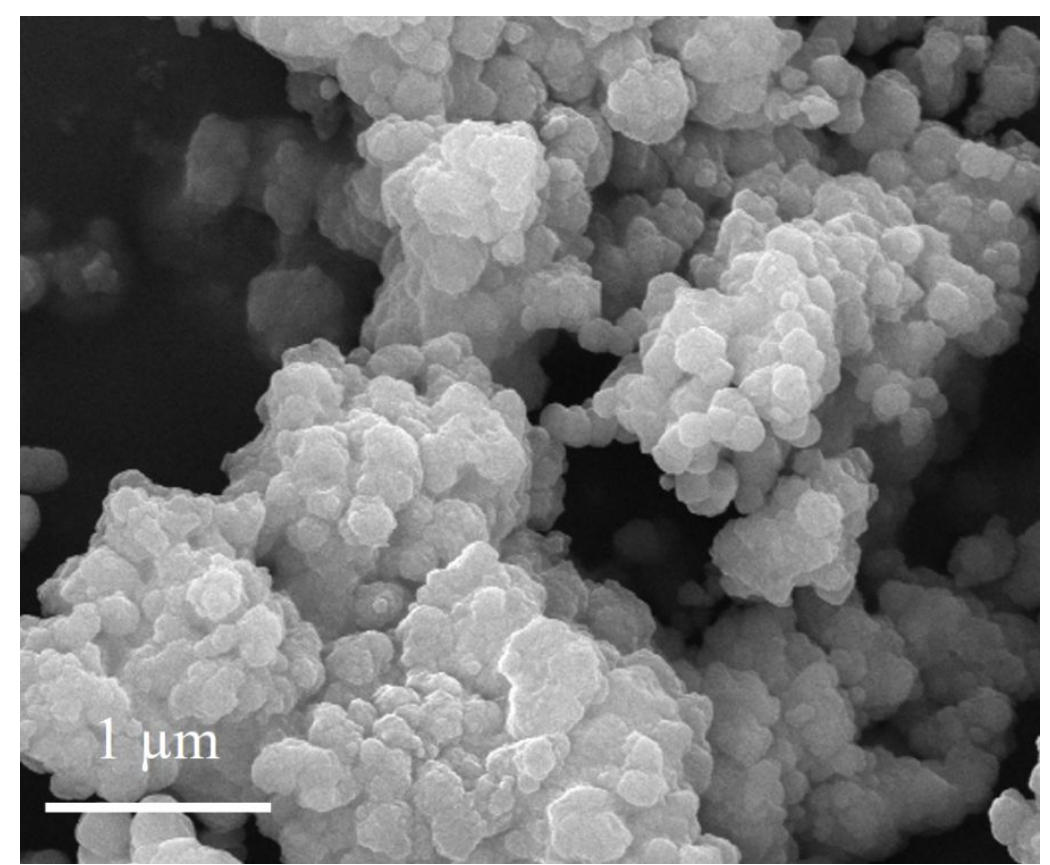

Figure S1. SEM photograph of UiO-66 MOFs. 


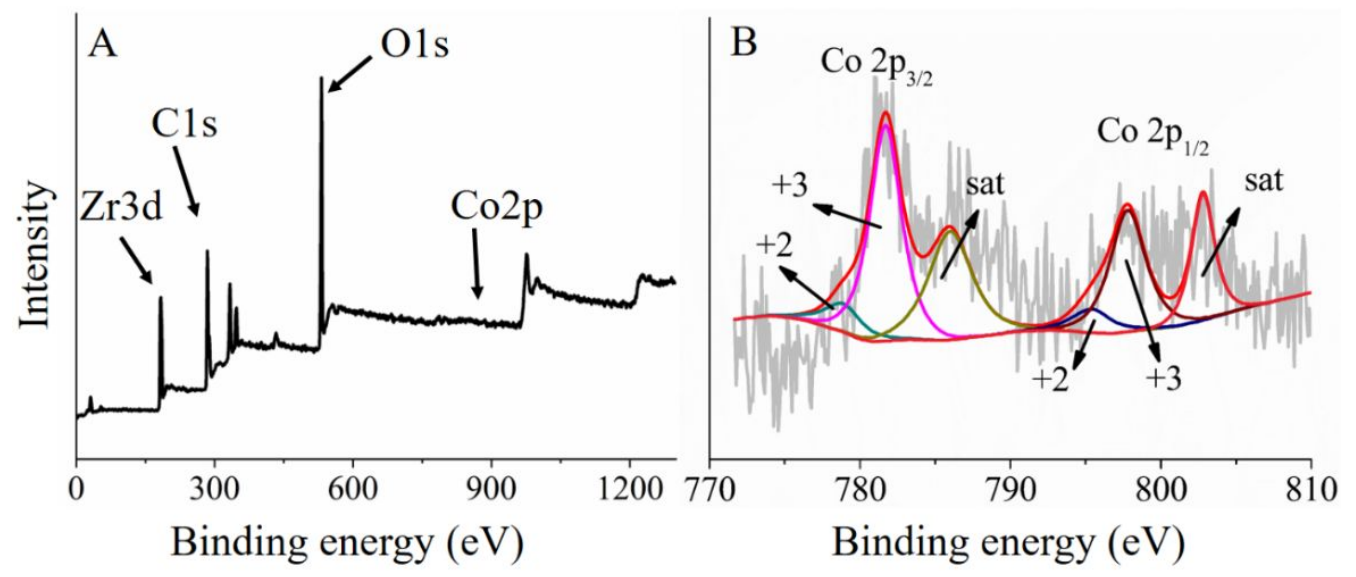

Figure S2. XPS patterns of Co SASCs: (A) survey and (B) Co 2p. 


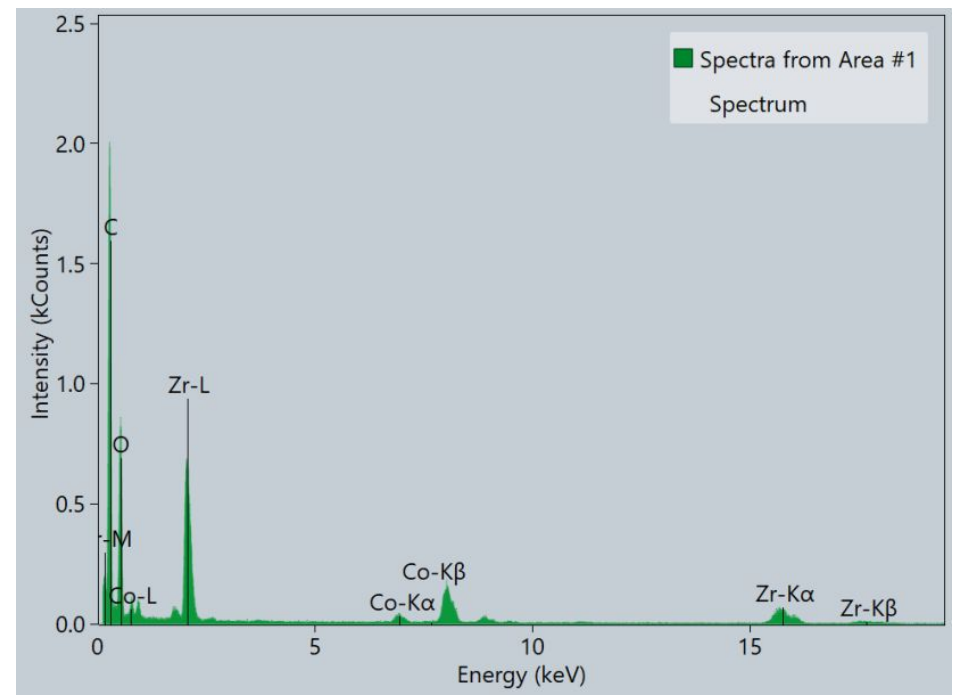

Figure S3. EDS spectrum of Co SASCs. 


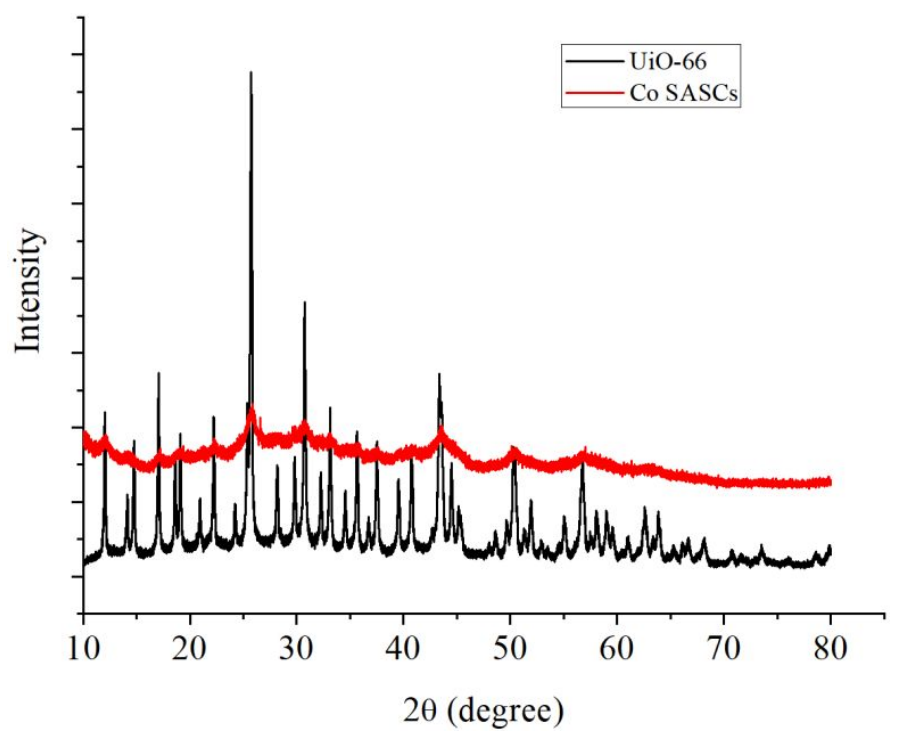

Figure S4. PXRD patterns of Co SASCs and UiO-66. 


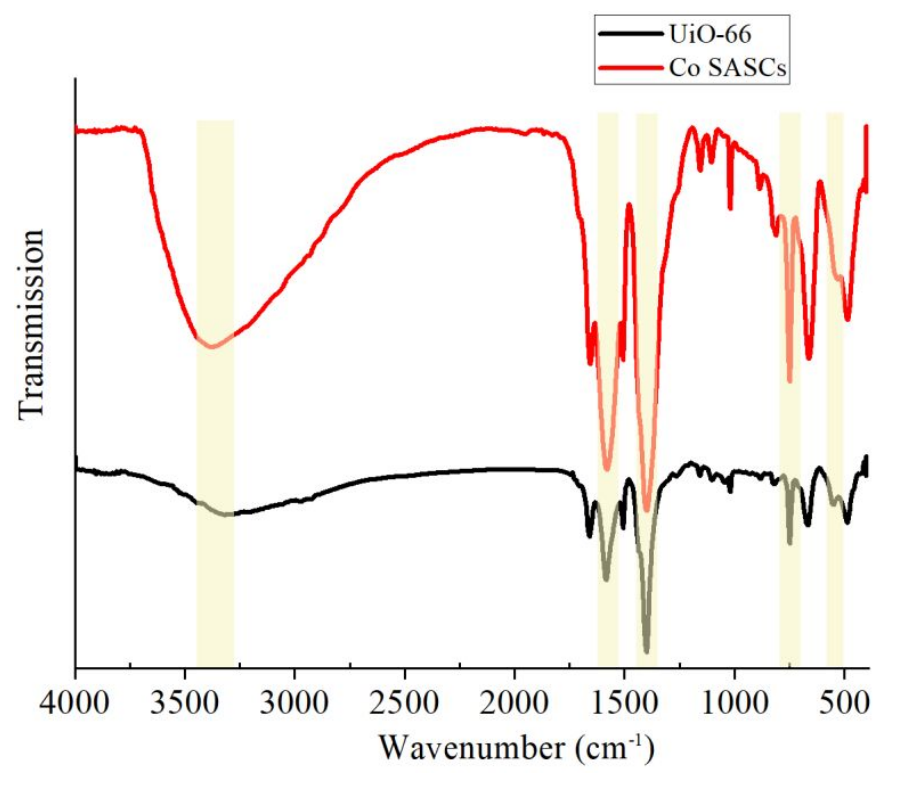

Figure S5. FTIR spectra of Co SASCs and UiO-66. The absorption peaks at $750 \mathrm{~cm}^{-1}, 1580$ $\mathrm{cm}^{-1}, 1380 \mathrm{~cm}^{-1}$ and $3440 \mathrm{~cm}^{-1}$ are attributed to the bending vibration of the $\mathrm{C}-\mathrm{H}$ bond on the benzene ring, the asymmetric vibration of the carboxyl group, the symmetric vibration of the carboxyl group, and the stretching vibration of the hydroxyl group, respectively. The absorption peak at $535 \mathrm{~cm}^{-1}$ is attributed to the stretching vibration of $\mathrm{Zr}-\mathrm{O} / \mathrm{Co}-\mathrm{O}$ bond. 

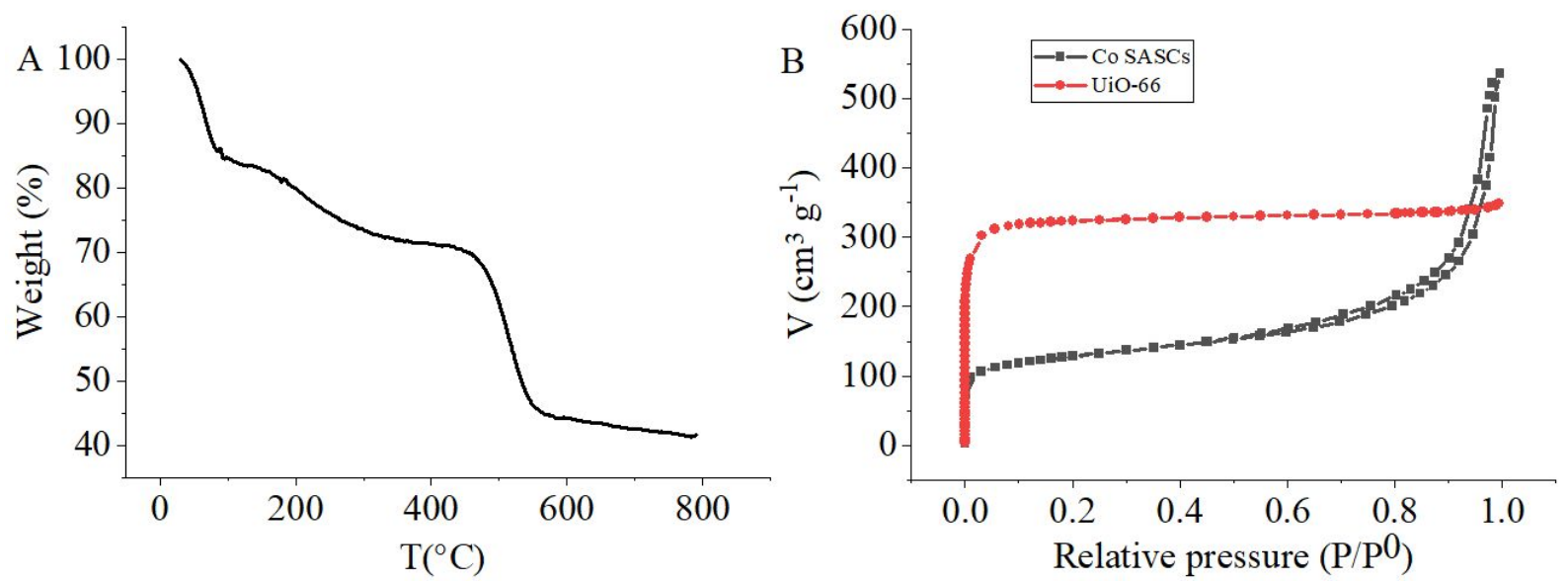

Figure S6. (A) Thermogravimetric analysis curve of Co SASCs measured in $\mathrm{N}_{2}$. (B) $\mathrm{N}_{2}$ adsorption/desorption isotherms of Co SASCs and UiO-66. 

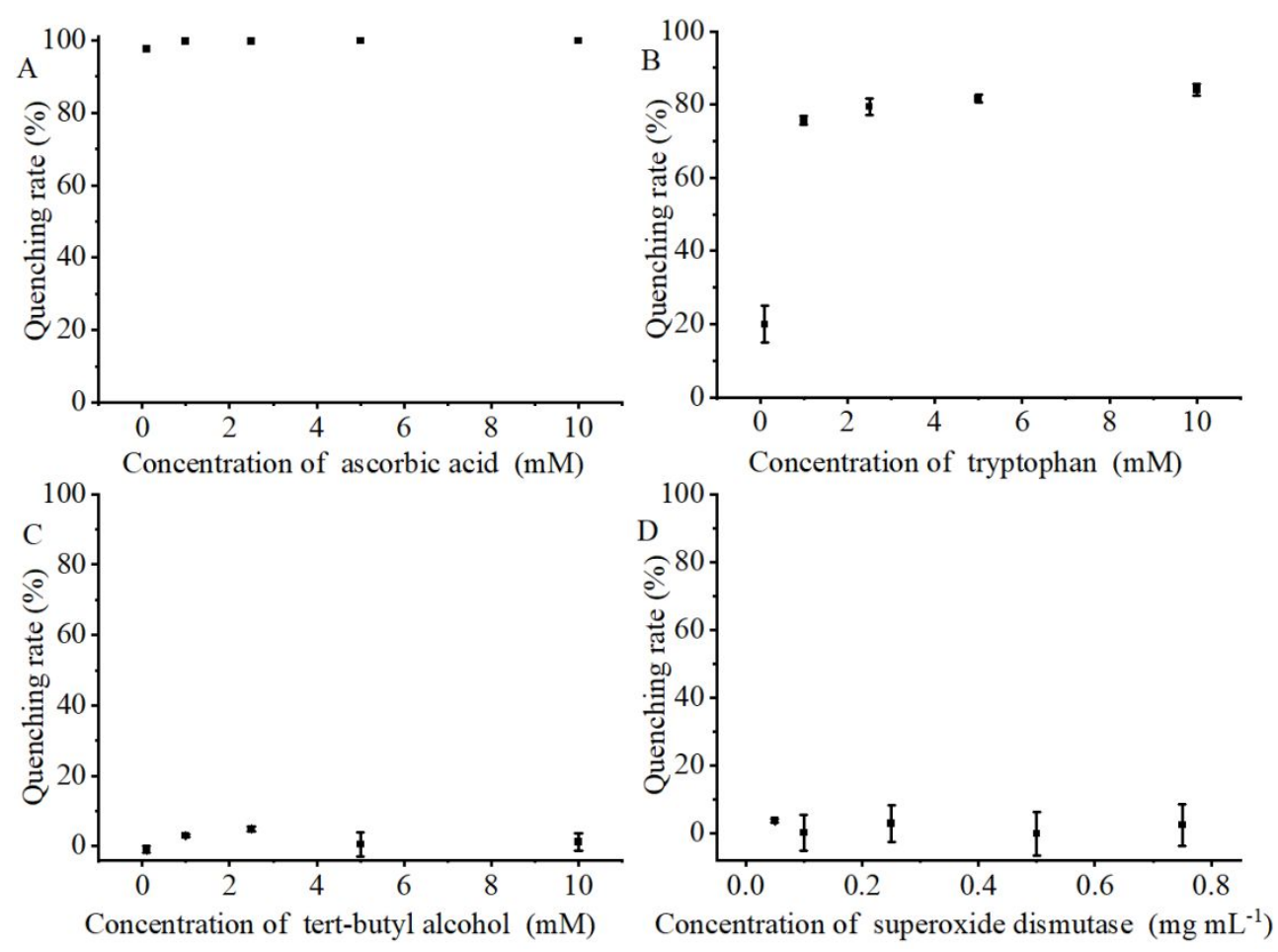

Figure S7. Quenching rates of different ROS scavengers on Co SASCs-enhanced luminol CL reaction: (A) ascorbic acid, (B) tryptophan, (C) tert-butyl alcohol and (D) superoxide dismutase $(n=3)$. 


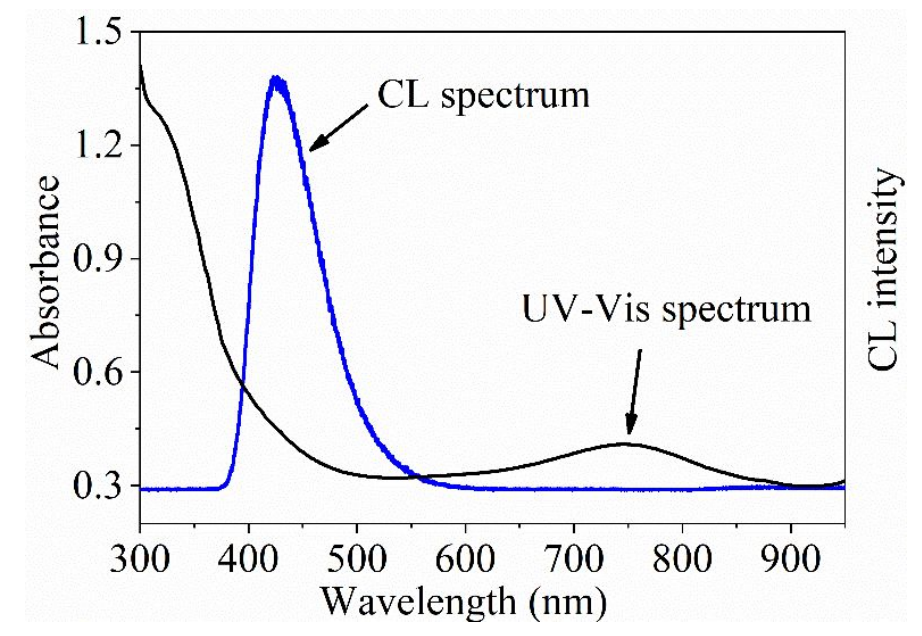

Figure S8. CL spectrum of Co SASCs-enhanced luminol- $\mathrm{H}_{2} \mathrm{O}_{2}$ reaction and absorption spectrum of $\mathrm{Ti}_{3} \mathrm{C}_{2}$ MXenes. 


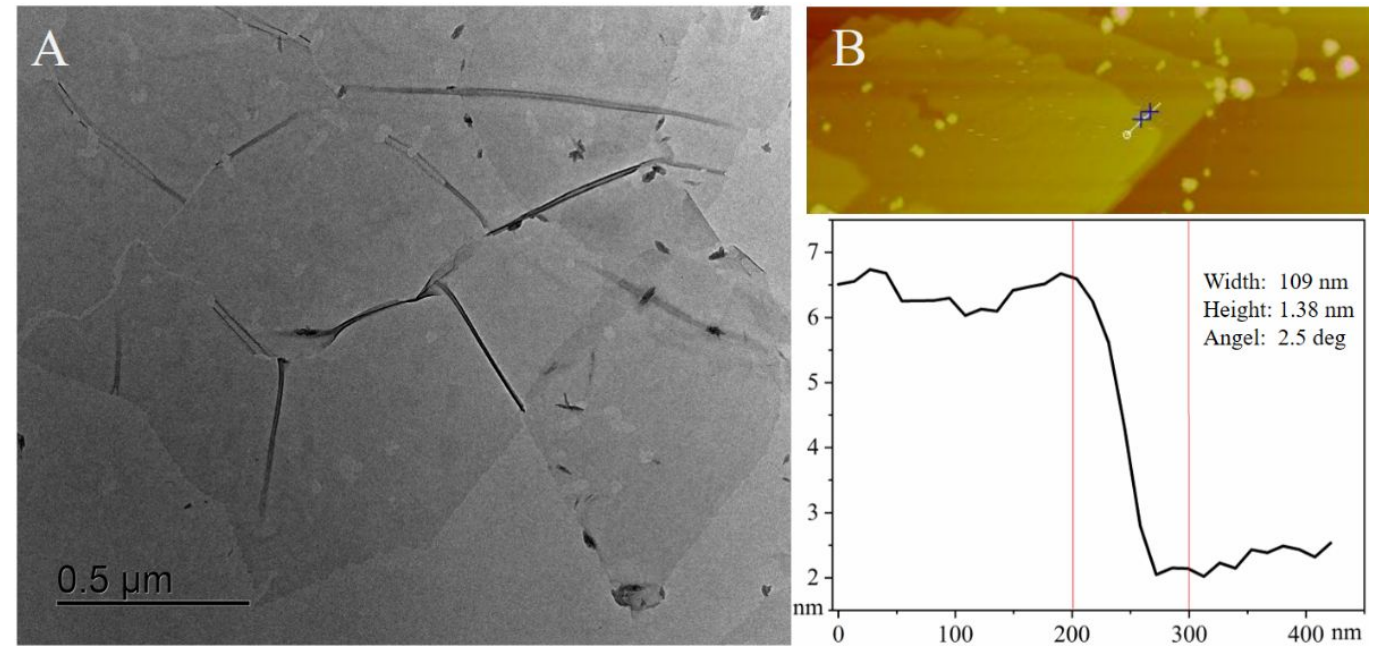

Figure S9. (A) TEM and (B) AFM photographs of $\mathrm{Ti}_{3} \mathrm{C}_{2}$ MXenes. 


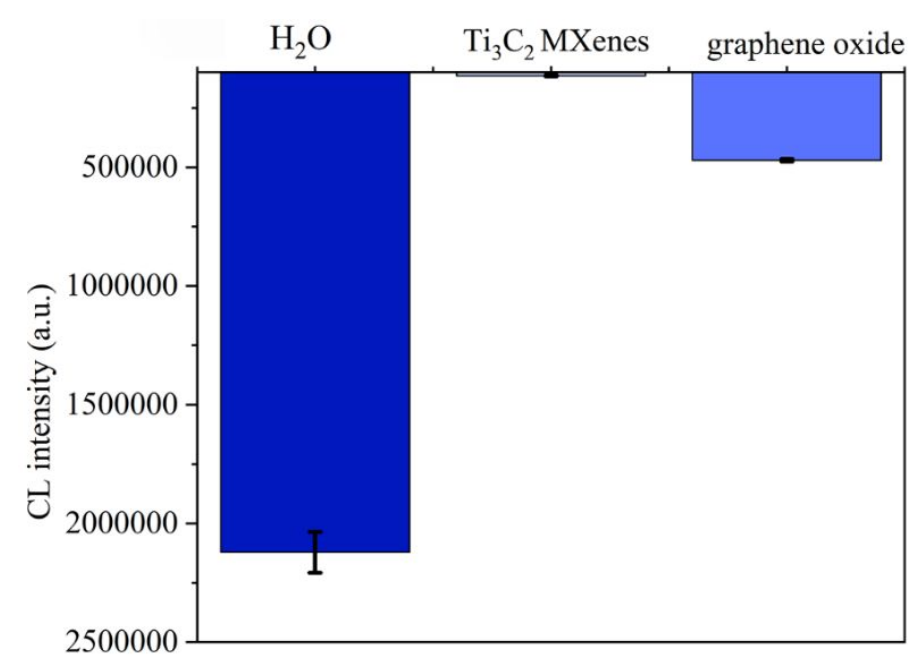

Figure S10. CL signals from Co SASCs-enhanced luminol reaction quenched by graphene oxide or monolayer $\mathrm{Ti}_{3} \mathrm{C}_{2}$ MXenes $(n=3)$. Here the both quenchers were at $100 \mu \mathrm{g} \mathrm{mL}^{-1}$, and $\mathrm{H}_{2} \mathrm{O}$ was adopted as a control. 

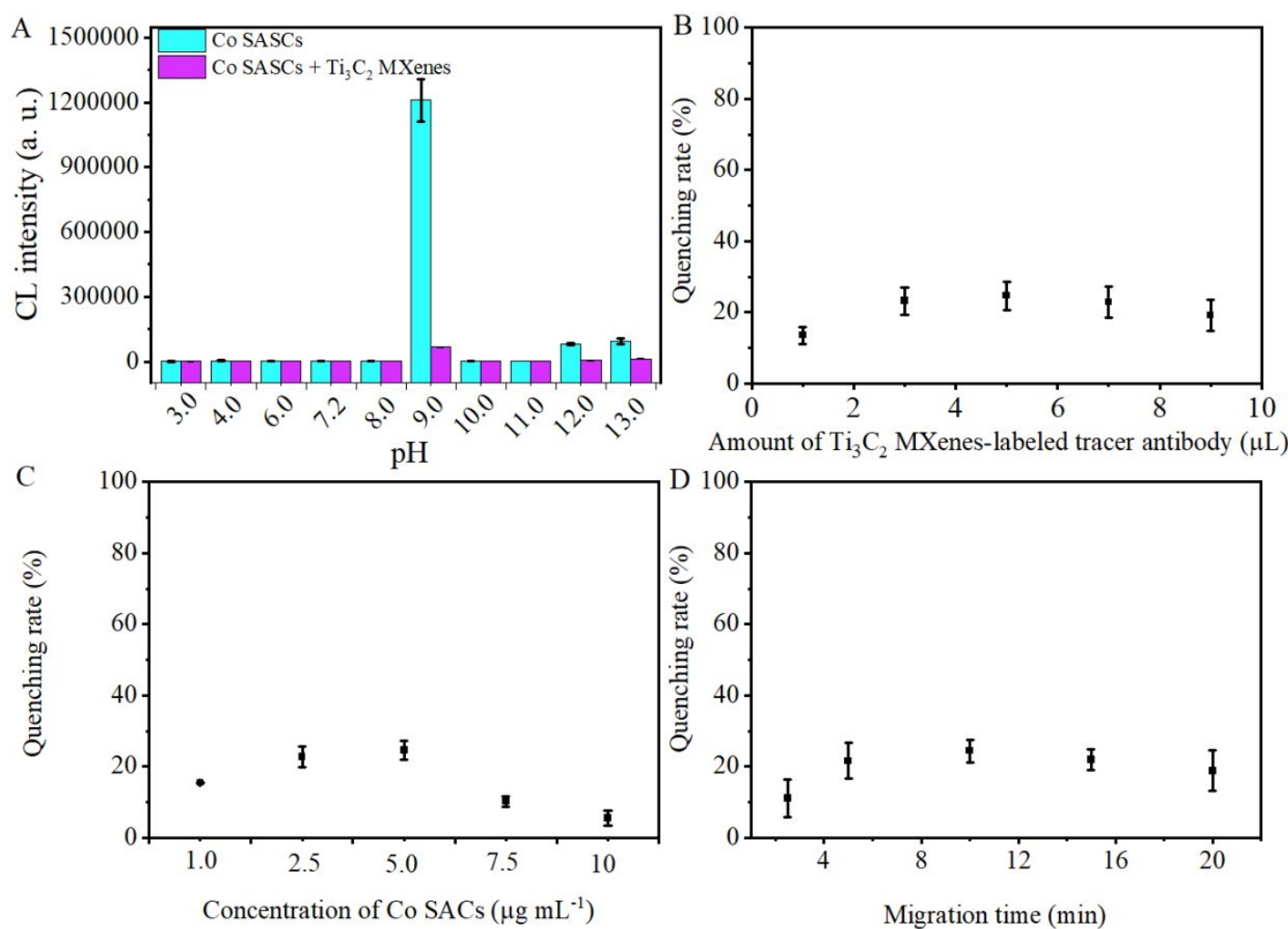

Figure S11. (A) Effect of $\mathrm{pH}$ value of the reaction medium on the CL signal from Co SASCsenhanced luminol reaction in the presence/absence of $\mathrm{Ti}_{3} \mathrm{C}_{2}$ Mxenes $(n=3)$. The concentration of luminol, $\mathrm{H}_{2} \mathrm{O}_{2}, \mathrm{Ti}_{3} \mathrm{C}_{2}$ MXenes and Co SASCs were $1.0 \times 10^{-6} \mathrm{M}, 0.1 \mathrm{M}, 50 \mu \mathrm{g} \mathrm{mL}^{-1}$, and 20 $\mu \mathrm{g} \mathrm{mL}-1$, respectively. (B) Effect of the amount of $\mathrm{Ti}_{3} \mathrm{C}_{2}$ MXenes-labeled tracer antibody on the CL signal for detecting cTnI at $7.5 \mathrm{pg} \mathrm{mL}^{-1}(n=3)$. (C) Effect of concentration of Co SASCs on the CL signal for detecting cTnI at $7.5 \mathrm{pg} \mathrm{mL}^{-1}(n=3)$. (D) Effect of migration time of $\mathrm{Ti}_{3} \mathrm{C}_{2}$ MXenes-labeled tracer antibody on the CL signal for detecting cTnI at $7.5 \mathrm{pg} \mathrm{mL}^{-1}(n=3)$. 


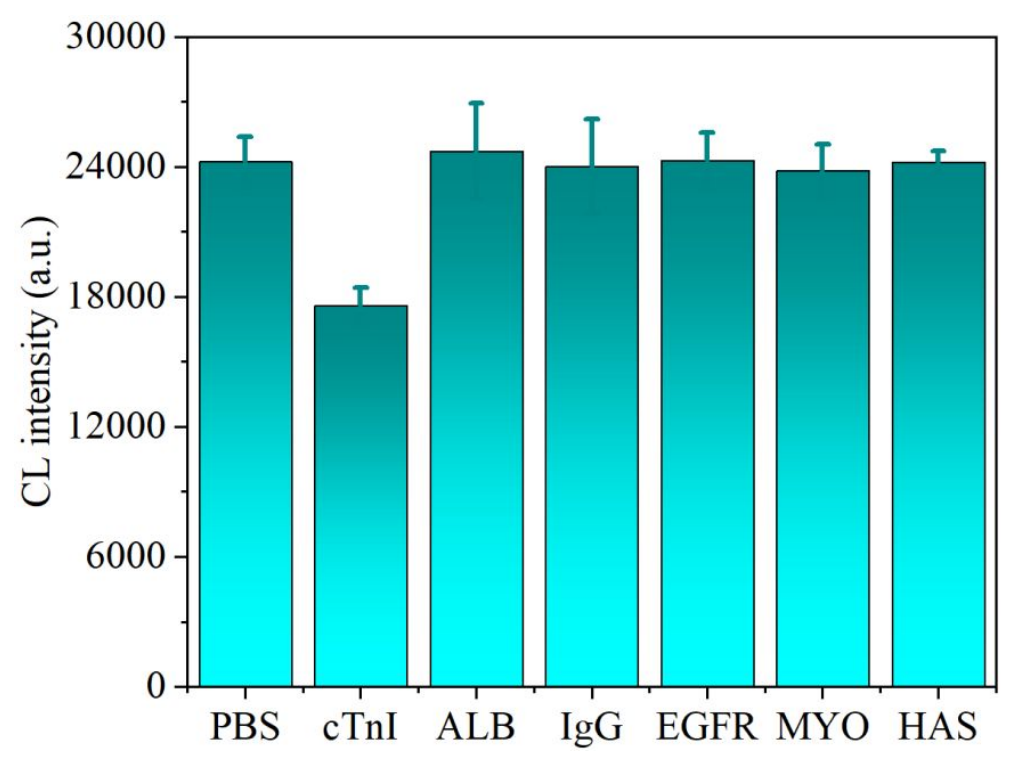

Figure S12. CL signals produced by cTnI and the potential interferents all at $10 \mathrm{pg} \mathrm{mL}^{-1}(n=$ $3)$. 
Table S1. Structural parameters of $\mathrm{Co}$ foil, $\mathrm{CoO}, \mathrm{Co}_{3} \mathrm{O}_{4}$, and $\mathrm{Co}$ SASCs extracted from the EXAFS fitting.

\begin{tabular}{|c|c|c|c|c|c|c|}
\hline & Path & $\mathrm{N}$ & $\Delta \mathrm{E}(\mathrm{eV})$ & $100 \times \mathrm{R}(\AA)$ & $100 \times \sigma^{2}\left(\AA^{2}\right)$ & R-factor \\
\hline \multirow{2}{*}{ Co foil } & $\mathrm{Co}-\mathrm{Co}$ & 12 & $5.94(0.56)$ & $249.4(0.3)$ & $6.28(0.43)$ & \multirow{2}{*}{0.00234} \\
\hline & Co-Col & 6 & $5.94(0.56)$ & $352.7(0.5)$ & $14.40(3.37)$ & \\
\hline \multirow{2}{*}{$\mathrm{CoO}$} & $\mathrm{Co}-\mathrm{O}$ & 6 & $-1.10(2.082)$ & 211.0(1.9) & $9.40(1.71)$ & \multirow{2}{*}{0.00765} \\
\hline & $\mathrm{Co}-\mathrm{Co}$ & 12 & $-3.42(1.194)$ & $300.2(0.9)$ & $10.22(0.90)$ & \\
\hline \multirow{3}{*}{$\mathrm{Co}_{3} \mathrm{O}_{4}$} & $\mathrm{Co}-\mathrm{O}$ & 5.33 & $-7.35(1.46)$ & $191.3(0.8)$ & $2.67(0.69)$ & \multirow{3}{*}{0.00523} \\
\hline & Co-Col & 4 & $-7.72(0.77)$ & $285.3(0.5)$ & $2.54(0.50)$ & \\
\hline & $\mathrm{Co}-\mathrm{Co} 2$ & 9.33 & $-7.72(0.77)$ & $358.8(0.7)$ & $6.10(0.62)$ & \\
\hline $\begin{array}{c}\text { Co } \\
\text { SASCs }\end{array}$ & $\mathrm{Co}-\mathrm{O}$ & $6.00(0.57)$ & $-6.25(1.07)$ & 204.3(1.7) & $10.69(1.59)$ & 0.011 \\
\hline
\end{tabular}


Table S2. Comparison of analytical performances from different quenching-type protocols for detecting cTnI.

\begin{tabular}{|c|c|c|c|c|}
\hline Donor & Quencher & Method & $\begin{array}{l}\text { Detection } \\
\text { limit }\end{array}$ & Reference \\
\hline FAM dye & Graphene oxide & Fluorescence & $\begin{array}{c}70 \\
\mathrm{pg} / \mathrm{mL}\end{array}$ & $\mathrm{S} 1$ \\
\hline $\operatorname{Ru}(\mathrm{dcbpy})_{3}{ }^{2+}$ & $\begin{array}{l}\mathrm{Au} / \text { carbon nitride } \\
\text { nanoposites }\end{array}$ & $\begin{array}{l}\text { Electrochemi- } \\
\text { luminesence }\end{array}$ & $\begin{array}{c}3.94 \\
\mathrm{fg} / \mathrm{mL}\end{array}$ & $\mathrm{S} 2$ \\
\hline FAM dye & Graphene oxide & Fluorescence & $\begin{array}{c}14.4 \\
\mathrm{pg} / \mathrm{mL}\end{array}$ & $\mathrm{S} 3$ \\
\hline $\begin{array}{c}\text { CdAgTe } \\
\text { quantum dots }\end{array}$ & Au nanoparticles & photoelectrochemistry & $\begin{array}{l}1.756 \\
\mathrm{pg} / \mathrm{mL}\end{array}$ & $\mathrm{S} 4$ \\
\hline $\begin{array}{l}\text { Europium } \\
\text { Si nanoparticle }\end{array}$ & Au nanorods & Fluorescence & $\begin{array}{l}97 \\
\mathrm{pg} / \mathrm{mL}\end{array}$ & S5 \\
\hline $\begin{array}{l}\text { Graphene } \\
\text { quantum dots }\end{array}$ & Graphene & Fluorescence & $\begin{array}{c}0.192 \\
\mathrm{pg} / \mathrm{mL}\end{array}$ & S6 \\
\hline Cy5 dye & $\begin{array}{c}\text { Magnetic } \\
\text { nanoparticles }\end{array}$ & Fluorescence & $\begin{array}{c}49 \\
\mathrm{pg} / \mathrm{mL}\end{array}$ & S7 \\
\hline BODIPY dye & Gold nanoparticles & Fluorescence & $\begin{array}{c}190 \\
\mathrm{pg} / \mathrm{mL}\end{array}$ & $\mathrm{S} 8$ \\
\hline $\begin{array}{c}\text { Graphene } \\
\text { quantum dots }\end{array}$ & Gold nanoclusters & $\begin{array}{l}\text { Electrochemi- } \\
\text { luminesence }\end{array}$ & $\begin{array}{l}0.354 \\
\mathrm{pg} / \mathrm{mL}\end{array}$ & S9 \\
\hline $\operatorname{Ru}(\mathrm{dcbpy})_{3}{ }^{2+}$ & $\mathrm{PtCu}_{3}$ Nanocubes & $\begin{array}{l}\text { Electrochemi- } \\
\text { luminesence }\end{array}$ & $12 \mathrm{fg} / \mathrm{mL}$ & $\mathrm{S} 10$ \\
\hline $\begin{array}{c}\text { Co SAC- } \\
\text { enhanced } \\
\text { luminol CL } \\
\text { reaction }\end{array}$ & $\mathrm{Ti}_{3} \mathrm{C}_{2}$ MXenes & $\mathrm{CL}$ & $0.33 \mathrm{pg} / \mathrm{mL}$ & $\begin{array}{l}\text { The } \\
\text { proposed } \\
\text { method }\end{array}$ \\
\hline
\end{tabular}




\section{References}

(S1) Liu, D. K.; Lu, X.; Yang, Y. W.; Zhai, Y. Y.; Zhang, J.; Li, L. A Novel Fluorescent Aptasensor for the Highly Sensitive and Selective Detection of Cardiac Troponin I Based on a Graphene Oxide Platform Anal. Bioanal. Chem. 2018, 410, 4285-4291.

(S2) Ye, J.; Zhu, L. P.; Yan, M. X.; Zhu, Q. J.; Lu, Q. Q.; Huang, J. S.; Cui, H.; Yang, X. R. Dual-Wavelength Ratiometric Electrochemiluminescence Immunosensor for Cardiac Troponin I Detection. Anal. Chem. 2019, 91, 1524-1531.

(S3) Li, Y; Dai, W. Q.; Lv, X. F.; Deng, Y. L. Aptamer-Based Rolling Circle Amplification Coupled with Graphene Oxide-Based Fluorescence Resonance Energy Transfer for Sensitive Detection of Cardiac Troponin I. Anal. Methods 2018, 10, 1767-1773.

(S4) Tan, Y.; Wang, Y. Y.; Li, M. S.; Ye, X. X.; Wu, T.; Li, C. Y. Enhanced Photoelectrochemical Immunosensing of Cardiac Troponin I Based on Energy Transfer between N-Acetyl-L-Cysteine Capped CdAgTe Quantum Dots and Dodecahedral Au Nanoparticles. Biosens. Bioelectron. 2017, 91, 741-746.

(S5) Lee, K.; Kim, K.; Chun, H.; Jeong, K.; Hong, D.; Lee, K.; Yoon, H. Time-Resolved Fluorescence Resonance Energy Transfer-Based Lateral Flow Immunoassay Using a Raspberry-Type Europium Particle and a Single Membrane for the Detection of Cardiac Troponin I. Biosens. Bioelectron. 2020, 163, 112284.

(S6) Bhatnagar, D.; Kumar, V.; Kumar, A.; Kaur, I. Graphene Quantum Qots FRET Based Sensor for Early Detection of Heart Attack in Human. Biosens. Bioelectron. 2016, 79, 495499.

(S7) Gong, X. Q.; Zhang, B.; Piao, J. F.; Zhao, Q.; Gao, W. C.; Peng, W. P.; Kang, Q.; Zhou, D. M.; Shu, G. M.; Chang, J. High Sensitive and Multiple Detection of Acute Myocardial Infarction Biomarkers Based on a Dual-Readout Immunochromatography Test Strip. Nanomed.: Nanotechnol., Biol. Med. 2018, 14, 1257-1266.

(S8) Kim, S.; Cho, I.; Park, J.; Seo, S.; Paek, S. A High-Performance Fluorescence Immunoassay Based on the Relaxation of Quenching, Exemplified by Detection of Cardiac Troponin I. Sensors 2016, 16, 669.

(S9) Wu, Z. L.; Liu, S. H.; Li, Y. Y.; Tang, F.; Zhao, Z. D.; Liu, Q.; Li, Y. Y.; Wei, Q. Electrochemiluminescence Resonance Energy Transfer System Fabricated by Quantum State Complexes for Cardiac Troponin I Detection. Sens. Actuators, B 2021, 336, 129733.

(S10) Zhang, L.; Xiong, C. Y.; Wang, H. J.; Yuan, R.; Chai, Y. Q. A Sensitive Electrochemiluminescence Immunosensor for Cardiac Troponin I Detection Based on Dual 
Quenching of the Self-Enhanced Ru (II) Complex by Folic Acid and In Situ Generated Oxygen. Sens. Actuators, B 2017, 241, 765-772. 